\title{
Parameterized Vertical-Axis Wind Turbine Wake Model Using CFD Vorticity Data
}

Andrew Ning

Brigham Young University, aning@byu.edu

Eric Tingey

Brigham Young University, ebtingey@gmail.com

Follow this and additional works at: https://scholarsarchive.byu.edu/facpub

Part of the Mechanical Engineering Commons

\section{Original Publication Citation}

Tingey, E., and Ning, A., "Parameterized Vertical-Axis Wind Turbine Wake Model Using CFD

Vorticity Data," ASME Wind Energy Symposium, San Diego, CA, Jan. 2016. doi:10.2514/

6.2016-1730

\section{BYU ScholarsArchive Citation}

Ning, Andrew and Tingey, Eric, "Parameterized Vertical-Axis Wind Turbine Wake Model Using CFD Vorticity Data" (2016). Faculty Publications. 1720.

https://scholarsarchive.byu.edu/facpub/1720

This Conference Paper is brought to you for free and open access by BYU ScholarsArchive. It has been accepted for inclusion in Faculty Publications by an authorized administrator of BYU ScholarsArchive. For more information, please contact ellen_amatangelo@byu.edu. 


\title{
Parameterized Vertical-Axis Wind Turbine Wake Model Using CFD Vorticity Data
}

\author{
Eric B. Tingey* and Andrew Ning ${ }^{\dagger}$ \\ Brigham Young University, Provo, Utah, 84602, USA
}

\begin{abstract}
In order to analyze or optimize a wind farm layout, reduced-order wake models are often used to estimate the interactions between turbines. While many such models exist for horizontal-axis wind turbines, for vertical-axis wind turbines (VAWTs) a simple parametric wake model does not exist. Using computational fluid dynamic (CFD) simulations we computed vorticity in a VAWT wake, and parameterized the data based on normalized downstream positions, tip-speed ratio, and solidity to predict a normalized wake velocity deficit. When compared to CFD, which takes about a day to run one simulation, the reduced-order model predicts the velocity deficit at any location within 5-6\% accuracy in a matter of milliseconds. The model was also found to agree well with trends observed in experimental data. Future additions will allow the reduced-order model to be used in wind farm layout analysis and optimization by accounting for multiple wake interactions.
\end{abstract}

\section{Nomenclature}

$D \quad$ turbine diameter

$F \quad$ sigmoid curve decay rate

I sigmoid curve inflection point

$M \quad$ sigmoid curve maximum value

$N \quad$ number of turbine blades

$R \quad$ turbine radius

$U_{\infty} \quad$ free stream velocity

$\alpha \quad$ EMG skew parameter

$\gamma \quad$ vorticity strength

$\kappa \quad$ EMG scale parameter

$\lambda \quad$ tip-speed ratio $=(\omega R) / U_{\infty}$

$\nu \quad$ EMG spread parameter

$\omega \quad$ rotation rate

$\sigma \quad$ solidity $=(N c) / R$

$\xi \quad$ EMG location parameter

$c \quad$ chord length

$u \quad$ velocity in the downstream direction

$x \quad$ downstream position

$x_{o} \quad$ specified downstream position for velocity calculation

$y \quad$ lateral position

$y_{o} \quad$ specified lateral position for velocity calculation

*Graduate Student, Department of Mechanical Engineering, AIAA Student Member

$\dagger$ Assistant Professor, Department of Mechanical Engineering, AIAA Senior Member 


\section{Introduction}

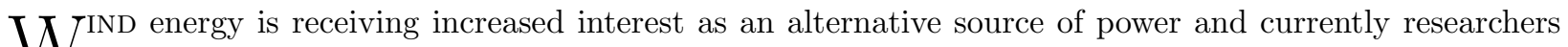
are pushing to move wind turbines to offshore locations where the wind is stronger and more consistent than land-based locations. ${ }^{1}$ However, one of the difficulties in using turbines in offshore locations is the large cost required to install and maintain turbines in ocean environments. ${ }^{2}$ Horizontal-axis wind turbines (HAWTs), the type of wind turbine generally used in large-scale land-based applications, are difficult to maintain because their drivetrain is located at the top of the tower and servicing it in offshore environments requires the use of expensive sea vessels. ${ }^{3}$ HAWTs also require yaw and pitch systems to align the turbine with the wind direction and regulate power, adding additional maintenance costs. ${ }^{4}$

Vertical-axis wind turbines (VAWTs) solve many of these challenges due to their simpler design and operation. The VAWT drivetrain can be located near the base affording easier access ${ }^{5}$ and VAWTs can be made smaller than HAWTs allowing more of them to be used in the same area as a HAWT wind farm, ${ }^{4}$ which could be beneficial for use in crowded urban environments. Additionally, VAWTs operate effectively no matter which way the wind is blowing, eliminating the need for a complex yaw system. These features make VAWTs a potential concept for better offshore and urban power production. ${ }^{5,6}$

However, a current problem with using VAWTs in large wind farm optimization is the lack of a simple model to predict how wakes propagate behind VAWTs. In the wake of a turbine, wind has less momentum and more turbulence which propagates downstream, potentially decreasing the power production of other turbines. ${ }^{7-9}$ When optimizing the cost of energy (total cost of the turbine divided by annual energy production) of many wind turbines in a wind farm, calculating the wake velocity deficit behind each turbine is done to determine the optimal wind farm layout. Using higher-fidelity modeling for these calculations, while producing accurate wake velocity results, could take large amounts of time to obtain results. This process takes even longer as the number of turbines increases with recalculations of the complex wake interactions done every time a turbine's position changes. Reduced-order wake models address this problem by parameterizing experimental wake behavior into simple mathematical models which can accurately predict the wake velocity deficits much more quickly than higher-fidelity modeling. Optimization of wind farm layouts with reduced-order wake models for HAWTs has been studied extensively, ${ }^{10-16}$ but this same type of optimization has not been done with VAWTs because a reduced-order wake model does not exist.

Although a wake model does not exist, there have been several studies involving the operation of VAWTs and the wakes they produce. In the 1970s and 1980s, Sandia National Laboratories conducted research comparing the performance of different types of VAWT designs. ${ }^{17-19}$ The focus of the research was to understand VAWT power output and efficiency, and their efforts resulted in a compilation of blade aerodynamic properties and power for different VAWT configurations. More recently, Delft University of Technology in the Netherlands conducted research based on the near wake formation of VAWTs using particle image velocimetry (PIV) which provided significant insight into the near wake development of VAWTs and a knowledge of contributing factors in turbine wake behavior. ${ }^{20-24}$ Shamsoddin and Porté-Agel also conducted wake research looking further downstream of a VAWT using large eddy simulation (LES) which showed good agreement between the experiments and LES models. ${ }^{25}$ While all of these efforts have been significant in helping us better understand the operation of VAWTs and how their wakes form, they have all been focused mainly on a specific VAWT configuration. However, large-scale wind turbine analysis requires parametric wake models that can predict a velocity profile based on different turbine parameters such as wind speed, rotation rate, and geometry.

Research at the California Institute of Technology proposed a wake model using a single point vortex and a doublet to simulate a VAWT (rotating cylinder flow). It also used simple expansion and decay models to predict the wake velocity distribution. ${ }^{26}$ While this was a good first step in modeling a VAWT wake using a simple model, it is not particularly accurate and is not able to be used in a generalized sense as it was tuned to a specific turbine. Therefore, the purpose of our research is to create a robust parametric VAWT wake model by studying the behavior of VAWT wakes over a large range of wind speeds, rotation rates, and geometries. The model will be developed to allow for the optimization of a wind farm layout using VAWTs in a reasonable amount of time. 


\section{CFD Modeling}

To produce a robust parametric wake model, flow around the turbine must be calculated based on varying conditions, such as the size of the turbine and how fast it rotates. Performing a wake analysis of a specific turbine limits a model's ability to calculate velocity accurately for a broad range of turbines. As our wake model was to be robust, we needed a large amount of wake data to predict how wakes propagate behind turbines at different wind speeds, rotation rates, and turbine geometries. We used computational fluid dynamics (CFD) software to simulate the turbines, as opposed to experimental procedures, as we needed a wide range of configurations. Using a CFD program called STAR-CCM+, we simulated an isolated VAWT with different tip-speed ratios (TSRs) and solidities using the unsteady 2D Reynolds-averaged Navier-Stokes equations, each which took about a day to compute. We modeled the VAWT in 2D rather than 3D as the fundamental process of energy conversion of a VAWT happens in the plane normal to the turbine's axis of rotation. ${ }^{21}$ While a $2 \mathrm{D}$ simulation does not account for finite blade effects, such as trailing and tip vortices, good agreement with experimental data was observed as noted in the validation studies.

Verification and validation of the CFD model is necessary to ensure that the model's cell size is refined enough and is producing results comparable to experimental data. For the verification, we performed a grid convergence study of a model based on a study of a straight-bladed VAWT conducted by Kjellin et al. ${ }^{27}$ We also performed a validation study using a PIV wake analysis conducted by Tescione et al. ${ }^{20}$

\section{A. CFD Mesh Verification}

In order to verify that the cell size of the CFD model was sufficiently refined, we performed a grid convergence study. To accomplish this, we modeled a turbine using the geometry specified by Kjellin et al. ${ }^{27}$ including a straight-bladed VAWT with a 6 meter diameter and three NACA 0021 blades, each with a chord length of 0.25 meters. The wind speed was set at $15 \mathrm{~m} / \mathrm{s}$ producing a Reynolds number of about 6,000,000. Keeping the TSR at 3.25 (at about the peak of performance from the experimental study), we ran simulations ranging from cell counts of about 400,000 to almost 5 million by reducing the base cell size of the CFD model by a factor of 1.4. The results of this grid convergence study can be seen in Fig. 1.

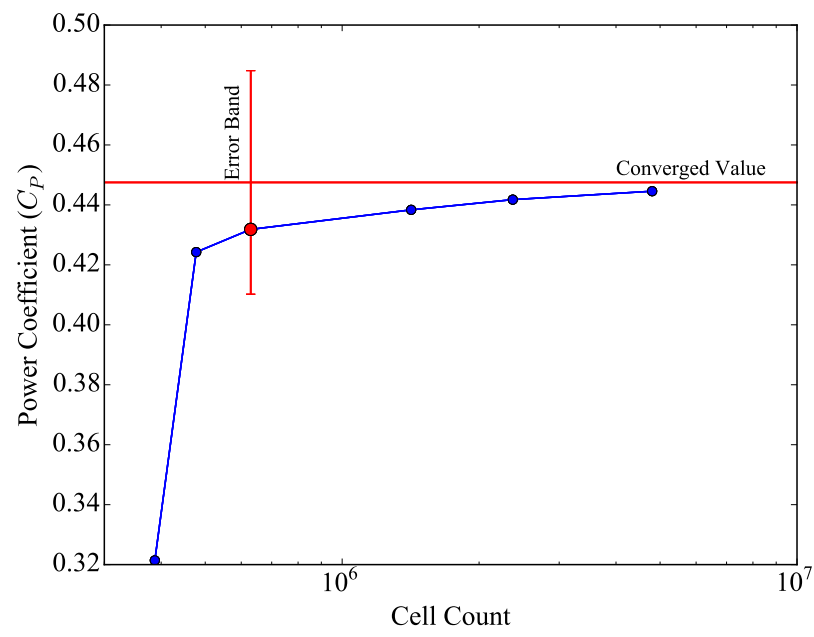

Figure 1. A plot of the grid convergence of the CFD model at a tip-speed ratio of 3.25. The converged value calculated with Richardson extrapolation is shown as well as the error band of the converged value. The model we used in our research is indicated by the red dot.

Using Richardson extrapolation, we concluded that the converged power coefficient was 0.458 with an error band of $3.73 \%$. In ideal circumstances, one would run the CFD models as refined as possible, but further refinement means more computational time for the CFD to reach a final solution. Therefore, a balance must be made between computational run time and sufficient refinement. In our case, we decided that about 630,000 cells was good enough for our CFD model as it produced a power coefficient of 0.432 (within the error band) while still running in a reasonable amount of time (this point is indicted by the red dot in Fig. 1). 


\section{B. Wake Velocity Validation}

We also validated the wake velocity of the CFD model to ensure that the CFD cell size was refined enough in the wake region. To do this, we used a study conducted by Tescione et al. ${ }^{20}$ in which a small turbine was tested in a wind tunnel at Delft University and velocities were measured with PIV. The turbine consisted of two straight NACA 0018 blades with a chord length of 0.06 meters. The turbine had a diameter of 1 meter and solidity of 0.24 . The VAWT was tested in a wind speed of $9.3 \mathrm{~m} / \mathrm{s}$, producing a Reynolds number of about 180,000, and run at a TSR of 4.5 .

The CFD model matched the PIV data well, as can be seen in Fig. 2, with the largest percent error of $23.0 \%$ of the maximum velocity deficit at $x / D=2.0$. This difference, as well as the asymmetry of the experimental data, could be due to the differences in experimental setup as opposed to ideal wind conditions in the CFD model. It is also reported that the asymmetry is caused by wake self-induction and stronger vorticity on one side. ${ }^{20}$ We concluded that the CFD model was sufficiently refined to use in obtaining VAWT wake data for our reduced-order model. This wake validation study also served as a point of reference for validating the reduced-order model as will be described later in the paper.
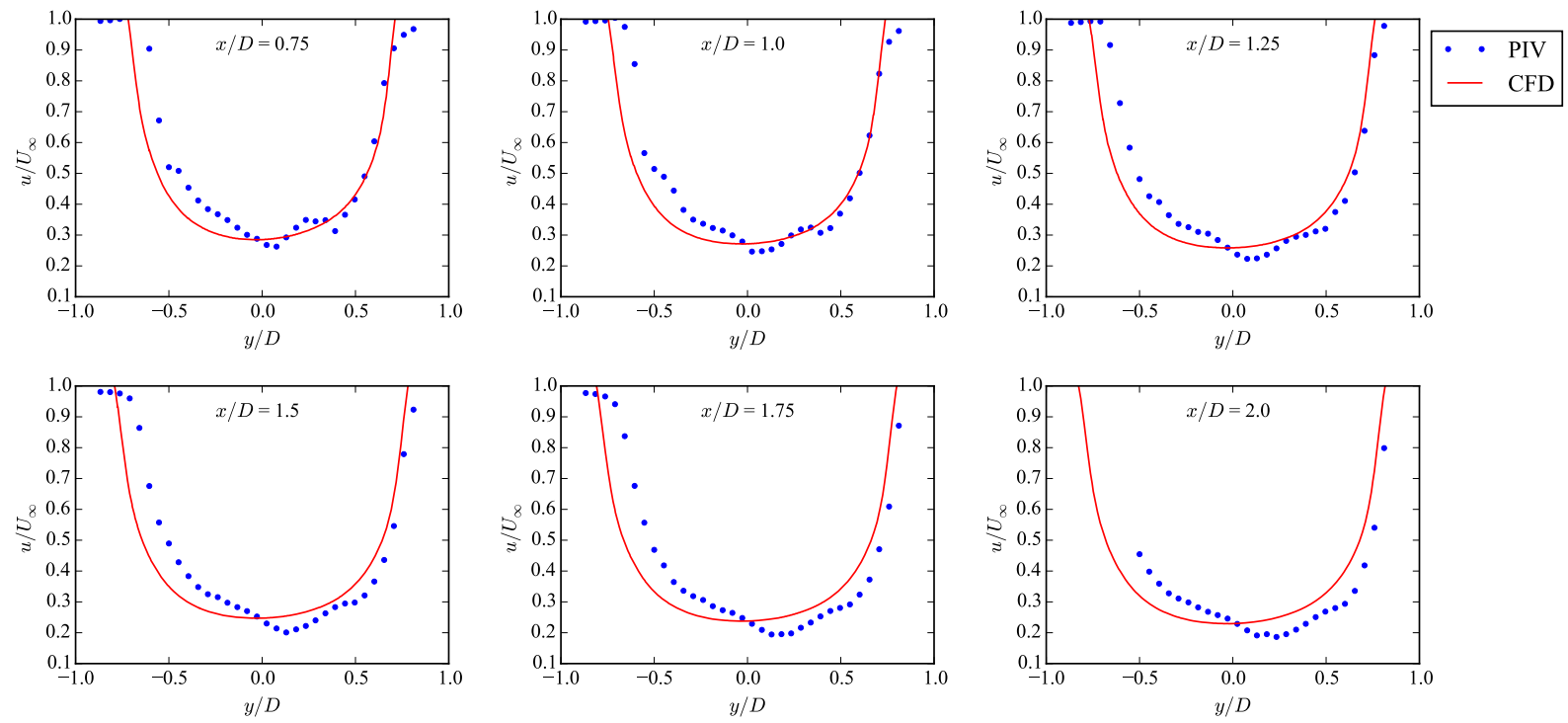

Figure 2. The wake velocity comparison between the PIV study conducted by Tescione et al. ${ }^{20}$ and our CFD model. The velocity $(u)$ is normalized by the free stream wind velocity $\left(U_{\infty}\right)$ and the downstream $(x)$ and lateral $(y)$ positions are normalized by the turbine diameter $(D)$.

\section{Reduced-Order Model Development}

The purpose of a reduced-order wake model is to simulate wake propagation accurately and quickly. In large-scale optimization, these benefits are used to produce results in a reasonable amount of time. As already shown, we were able to use CFD modeling to calculate the velocity in a VAWT wake accurately in about a day. However, when many turbines are involved and moving them to a new position requires new calculations, large-scale applications of this method become very time-consuming. Therefore, the performance of reducedorder wake models are an improvement to a CFD analysis when analyzing large amounts of turbines.

For our research, we used the CFD models for different VAWT simulations at TSRs ranging from 2.5 to 7.0 and solidities ranging from 0.15 to 1.0 based on the turbine diameter, airfoil shape, and Reynolds number described by Kjellin et al. ${ }^{27}$ and each CFD simulation took about a day to compute the results. However, as can be seen in Fig. 3(a), trying to capture trends of the CFD velocity profiles with numerical models would be very complicated as it would require parametrically modeling the velocity profile in the fluid domain both inside and outside of the wake region. Therefore, we decided to use the vorticity data as seen in Fig. 3(b) with its concentrated streams which can be parametrically modeled easily. The vorticity 


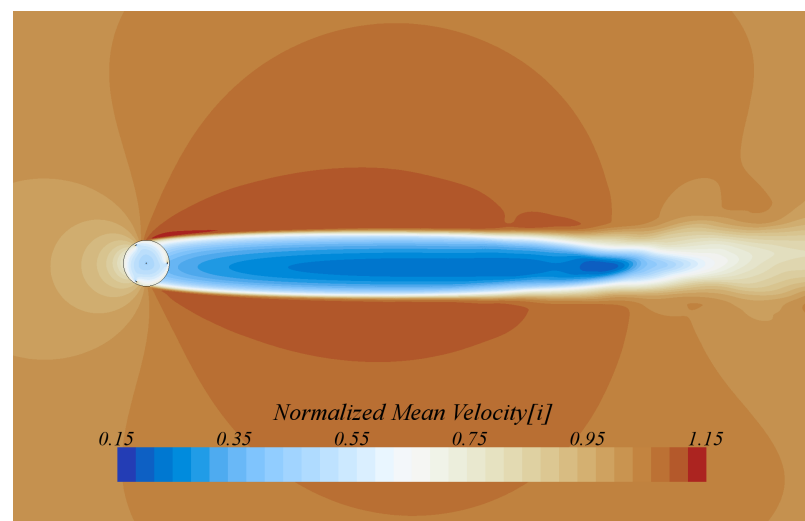

(a) CFD time-averaged velocity field

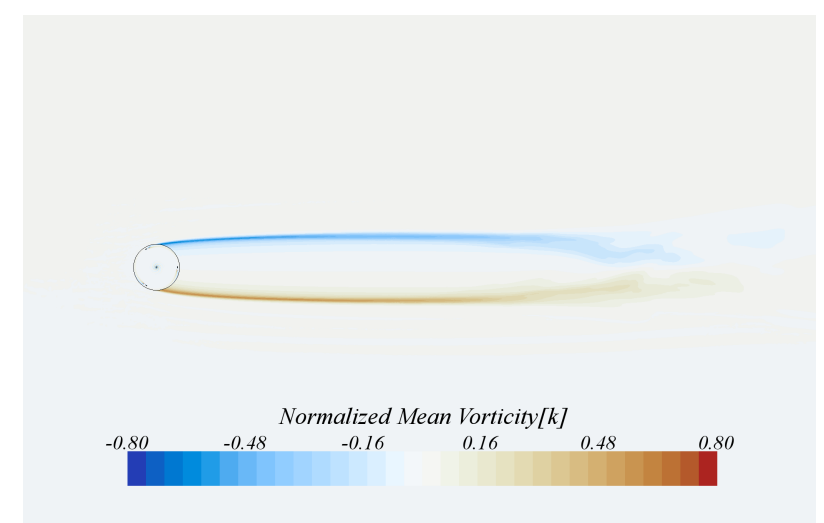

(b) CFD time-averaged vorticity field

Figure 3. Examples of the time-averaged velocity and vorticity fields found in the CFD model at a TSR of 4.5 and a solidity of 0.25 . The velocity is normalized by the free stream velocity at this TSR of $8.87 \mathrm{~m} / \mathrm{s}$ and the vorticity is normalized by the rotation rate of $13.3 \mathrm{rad} / \mathrm{s}$.

data was then used to reconstruct the velocity field using fluid dynamics.

Although the vorticity data is made up of concentrated streams, one can see in Fig. 3(b) that the vorticity comes to a maximum value at the center of the stream and then decays away quickly. To analyze this behavior numerically, we took 30 lateral cuts over the length of the domain, separated the cuts into positive and negative vorticity sections, and trend fitted each half separately with probabilistic distributions (see Fig. 4 for a representation of this process). To generalize the results for all turbines, both the lateral and downstream distances were normalized by the turbine diameter and the vorticity was normalized by the turbine's rotation rate.

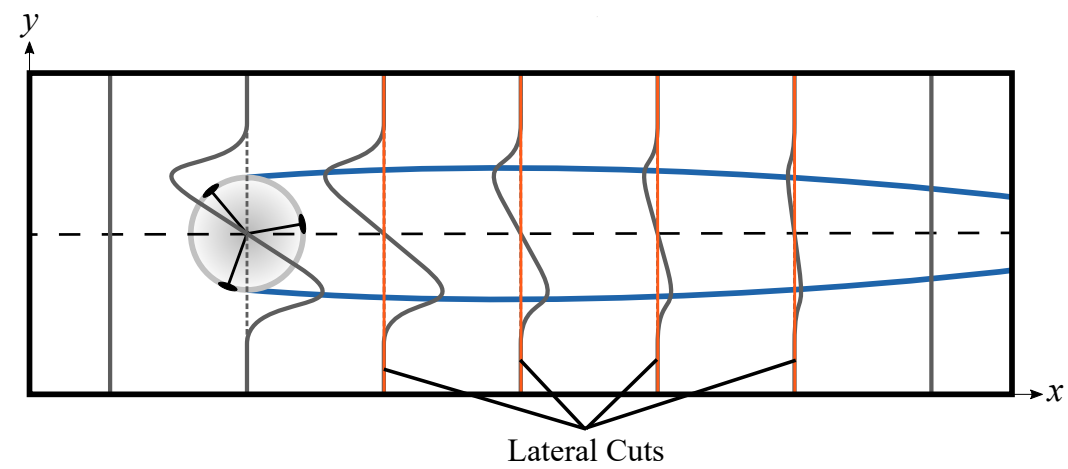

Figure 4. The lateral vorticity cuts made to analyze the vorticity data numerically seen in orange. An exaggerated representation of the vorticity distribution is shown with the gray lines. Each of the cuts were split into two sections for analysis based on where the vorticity changed sign (negative to positive) which was roughly at the center line. The downstream $(x)$ and lateral $(y)$ positions are normalized by the turbine diameter $(D)$ and the vorticity is normalized by the rotation rate $(\omega)$.

After exploring several different skewed probabilistic distributions, such as the skewed normal and Weibull distributions, we found that the best fit was attained using the exponentially modified Gaussian (EMG) distribution. This distribution is defined by four parameters: the location ( $\xi$; the position of the peak), the spread ( $\nu$; how wide or narrow the peak was), the skew ( $\alpha$; amount of skewness of the distribution), and the scale $(\kappa ;$ how high the peak reached). These parameters were used to calculate the vorticity strength $(\gamma(y / D))$ as a function of normalized lateral position $(y / D)$ in the equation:

$$
\gamma(y / D)=\kappa\left[\frac{\alpha}{2} \exp \left(\frac{\alpha}{2}\left(2 \xi+\alpha \nu^{2}-2(y / D)\right)\right) \operatorname{erf}\left(\frac{\xi+\alpha \nu^{2}-(y / D)}{\sqrt{2} \nu}\right)\right]
$$


Each of the lateral cuts were fitted with the EMG distribution and an example of positive vorticity cuts can be seen in Fig. 5.

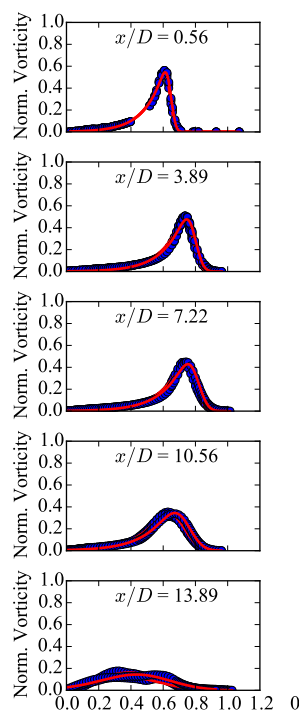

$y / D$
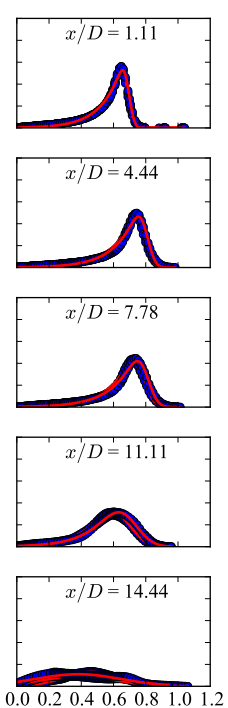

$y / D$
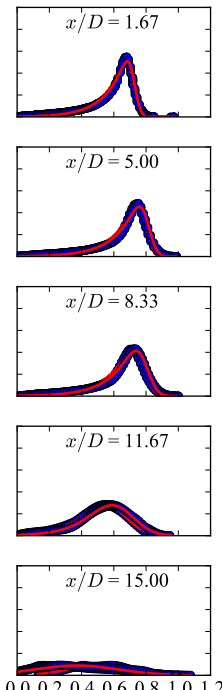

$y / D$
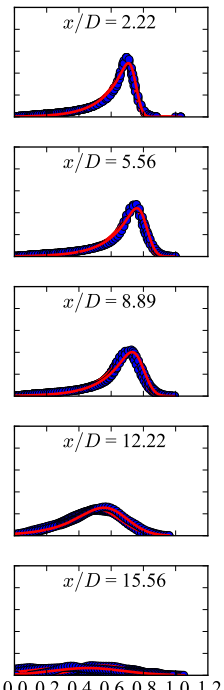

$y / D$
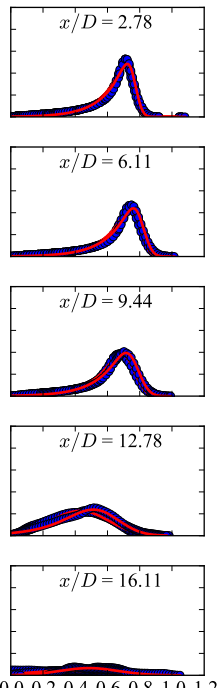

$y / D$
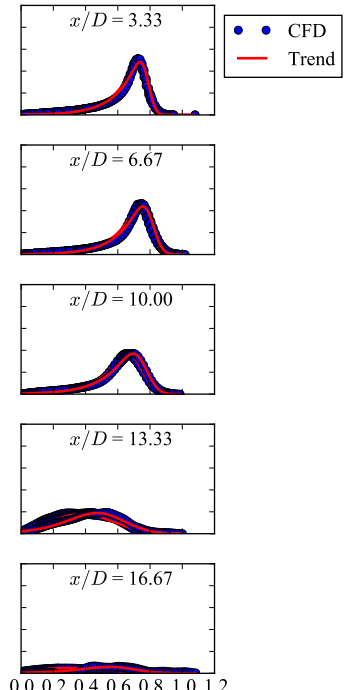

$y / D$

Figure 5. An example of positive lateral vorticity cuts from the center of the turbine wake going out to one side showing the CFD data compared to the trend fit. The vorticity is normalized by the rotation rate $(\omega)$ and the downstream $(x)$ and lateral $(y)$ positions are normalized by the turbine diameter $(D)$ at each cut.

After all of the vorticity data was fit with the EMG distribution, each of the four parameters were plotted over the normalized downstream distance, as seen in Fig. 6. The location data was fit with a quadratic curve (cyan and magenta lines in Fig. 6(a)) and the spread and skew data were fit with linear curves (cyan and magenta lines in Fig. 6(b) and (c)). Because the data became increasingly turbulent and random as the the vorticity decayed, the curve fits were only applied until the location where spread data sharply increased or decreased and the skew data became increasingly spread out. From the average of these downstream locations to the end of the data set, the turbulent EMG parameter data was neglected. We made this decision as the vorticity strength decays rapidly after its onset and trying to accurately capture the spread and skew was not necessary to predict the vorticity profile.

In an effort to make the trend fitting as accurate as possible, we calculated the total vorticity strength of each lateral cut using integration. Because the unscaled EMG distribution has an area of one, the scale parameter, $\kappa$, corresponds to the total vorticity strength (the area under the vorticity data curve). Instead of letting $\kappa$ be a free parameter in the trend fitting, we set it to the value of the calculated total vorticity strength and let the other parameters adjust to fit the shape of the curve. The scale data was then fit with a sigmoid curve defined by:

$$
S(x / D)=\frac{M}{(1+\exp (F((x / D)-I)))}+Y
$$

The sigmoid curve can be translated up or down by $Y$ to limit the decay to a specific value, but in our case we allowed the vorticity to decay to zero $(Y=0)$. This curve fit can be seen in Fig. 6(d) by the cyan and magenta lines.

We took averages of each of the curve fits for each side of the vorticity and plotted it in Fig. 6 with dotted black lines. Because the data was observed to be nearly symmetric, we assumed our model to be fully symmetric in order to reduce the number of parameters by half. In making the assumption of symmetry rather than using actual values, we effectively forced the final velocity profile to be symmetric as well. While this did not pose significant problems in velocity calculation, we did see some slight variations between the CFD data and the reduced-order model, which we will discuss later on. 
The quadratic, linear, and sigmoid curve fits all together resulted in 10 variables needed to define the wake vorticity as a function of the normalized downstream distance. This same process was repeated 95 times over the range of TSRs (2.5 to 7.0) and solidities (0.15 to 1.0) to produce a database of vorticity measurements as functions of TSR and solidity. As these vorticity measurements were made at discrete TSR and solidity values, we used a rectangular bivariate spline to interpolate the values we did not calculate. This allowed our vorticity database to be used for any TSR and solidity within the calculated range.

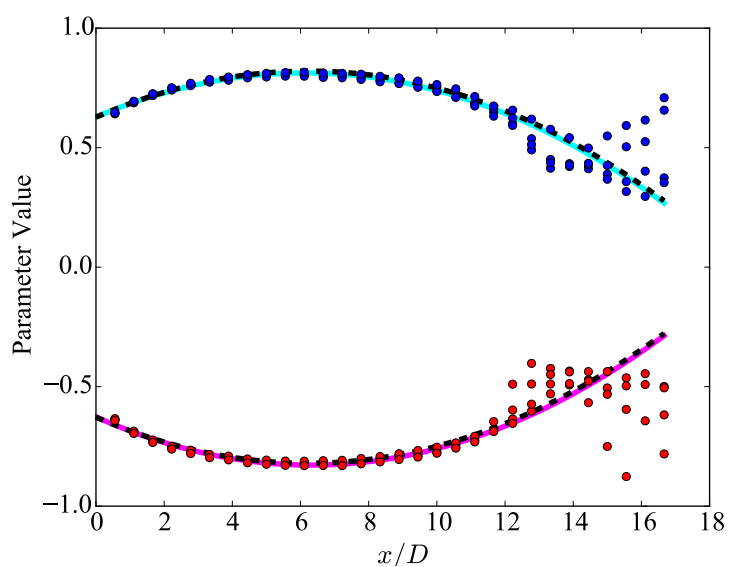

(a) Location Parameter $\xi$

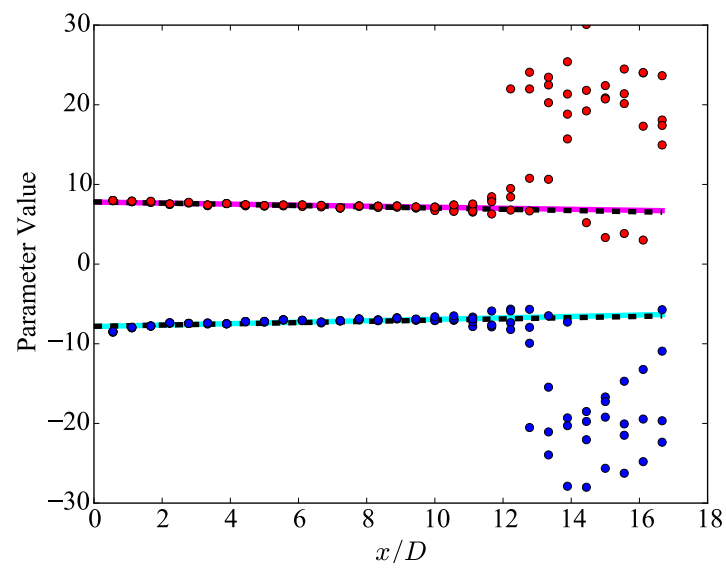

(c) Skew Parameter $\alpha$

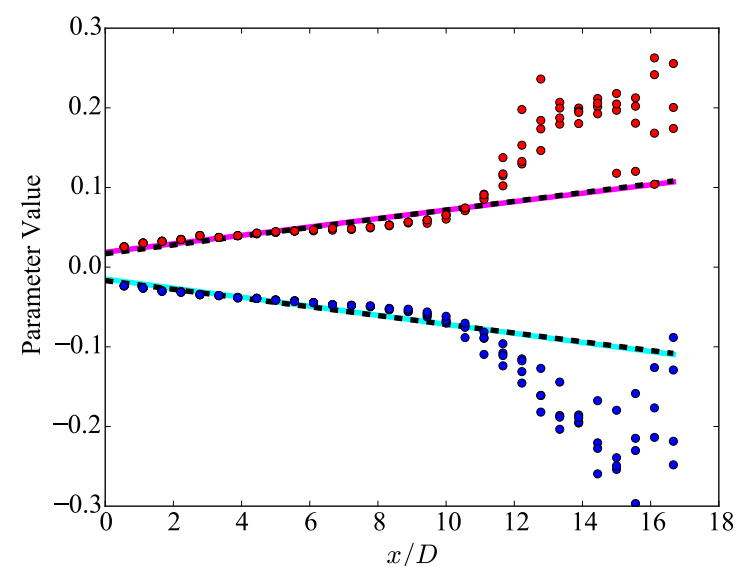

(b) Spread Parameter $\nu$

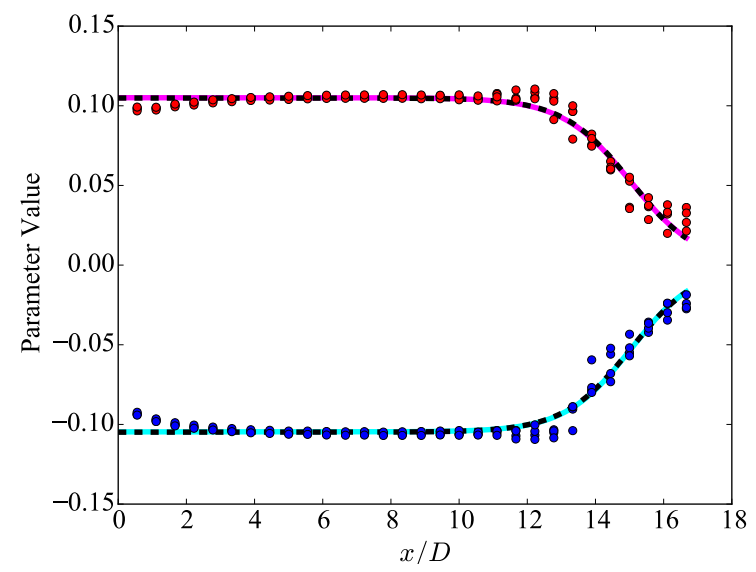

(d) Scale Parameter $\kappa$

Figure 6. The different parameters used in the EMG distribution fitting of the lateral vorticity cuts. The blue and red dots represent the values found from the CFD data trend fitting, cyan and magenta lines represent quadratic, linear, and sigmoid curve fits of each data set and the dotted black line represents an average of the two (showing near symmetry in the data). The downstream $(x)$ position is normalized by the turbine diameter $(D)$. The spreading of data points near the far downstream end is caused by increasing turbulence.

Using the parameterized vorticity measurements in both the downstream $(x)$ and lateral $(y)$ directions, we computed the velocity profile with the equation:

$$
u\left(x_{o}, y_{o}\right)=U_{\infty}+\left[\int_{a}^{b} \int_{-w}^{w} \frac{\gamma(x, y, \lambda, \sigma)}{2 \pi}\left[\frac{y-y_{o}}{\left(x-x_{o}\right)^{2}+\left(y-y_{o}\right)^{2}}\right] d y d x\right]
$$

The whole equation is integrated both in the lateral direction from $-w$ to $w$ and in the downstream direction from $a$ to $b$. These distances were based on where the vorticity had fully decayed, which we found to be 4 diameters laterally ( $-w$ and $w$ ) and 35 diameters downstream ( $a$ to $b$ ). Because this model was made to be generalized for all turbines, the length values are normalized by the turbine diameter, the vorticity is 
normalized by the turbine's rotation rate, and the velocity is normalized by the free stream wind speed in the final wake model. Fig. 7 shows an illustration of the variables used in this equation.

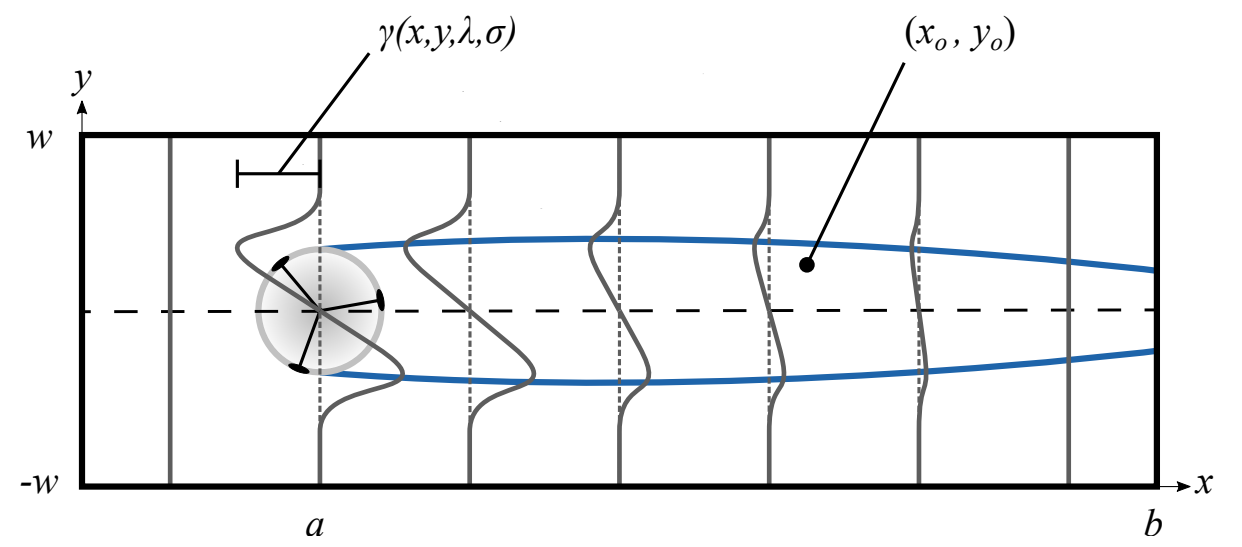

Figure 7. The vorticity profile behind a VAWT as defined by Eq. 3 with the vorticity magnitude shown by the gray lines.

\section{Results}

Using our developed reduced-order VAWT wake model, we compared the model's calculated velocity deficit with the CFD data and experimental results. These were done to ensure the model's calculations produced reasonable results compared to higher-fidelity methods. As shown in Fig. 8, the greatest difference between the CFD data and the reduced-order model occurs closer to the turbine. This is due to the CFD data deviating slightly from the scale parameter data of the EMG distribution close to the turbine (see Fig. 6(d)) causing the reduced-order model to predict a slightly lower vorticity strength. We plan to investigate this effect further and improve our wake model to account for the difference in vorticity, but overall the reducedorder model predicted a velocity deficit with a percent error of $5-6 \%$ of the CFD data neglecting the near turbine data. This quality shows very good comparison and confidence in using the reduced-order model in wind farm layout optimization applications.

Another difference that can be seen between the CFD data and the reduced-order model is that the reduced-order model has a slight flattening near the center as opposed the the CFD's rounded profile. One issue that was encountered when fitting the EMG curve to the CFD data was that near a $y / D$ of 0.0 , the EMG curve went to a normalized vorticity of 0.0 slightly faster than the CFD data closer to the turbine (see Fig. 5). Because of this behavior, less vorticity than is actually present was predicted causing the slight flattening in the velocity profile. From the trends we observed in wake decay and expansion, we found that parameterizing the vorticity using an EMG distribution was the best way to capture the CFD vorticity data. While the EMG distribution was not perfect, it produced reasonable velocity results.

As mentioned above, we assumed a symmetric vorticity distribution in order to eliminate variables needed in our final wake model and allow it to be simpler. As a result, the wake model produced a symmetric velocity deficit while the CFD tended to become slightly asymmetric further downstream of the VAWT (as seen especially in Fig. 8(f)). We plan to look into the affect of symmetry on the VAWT wake velocity results to see how much of an impact that error has on large-scale VAWT analysis, but for now we determined that the simplicity of symmetric velocity deficits outweighed the need for the complexity of asymmetry. 


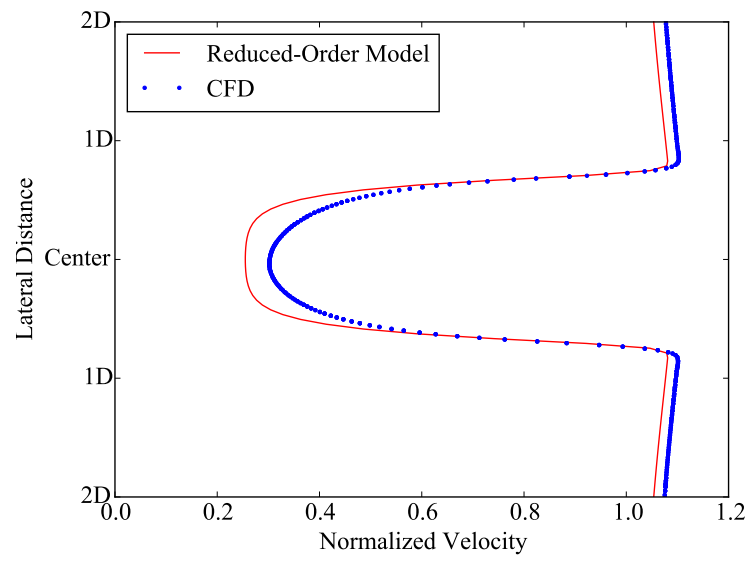

(a) $x / D=2.0 ; 15.54 \%$

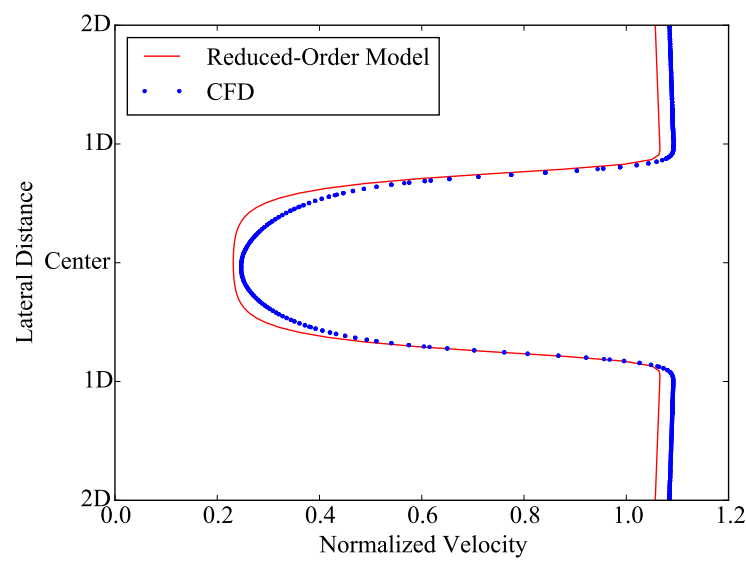

(c) $x / D=6.0 ; 6.27 \%$

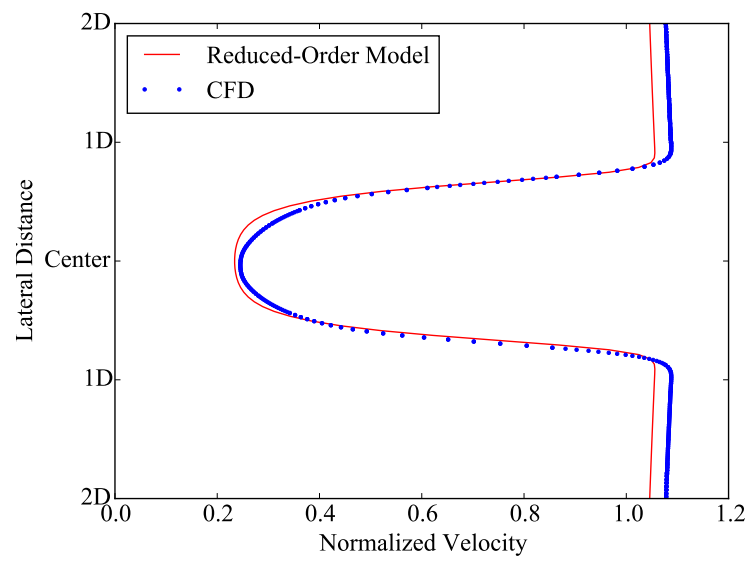

(e) $x / D=10.0 ; 4.57 \%$

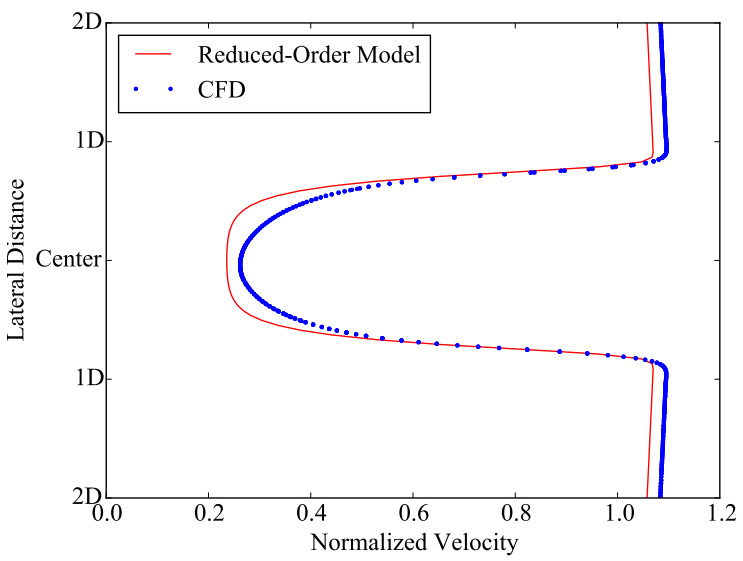

(b) $x / D=4.0 ; 9.97 \%$

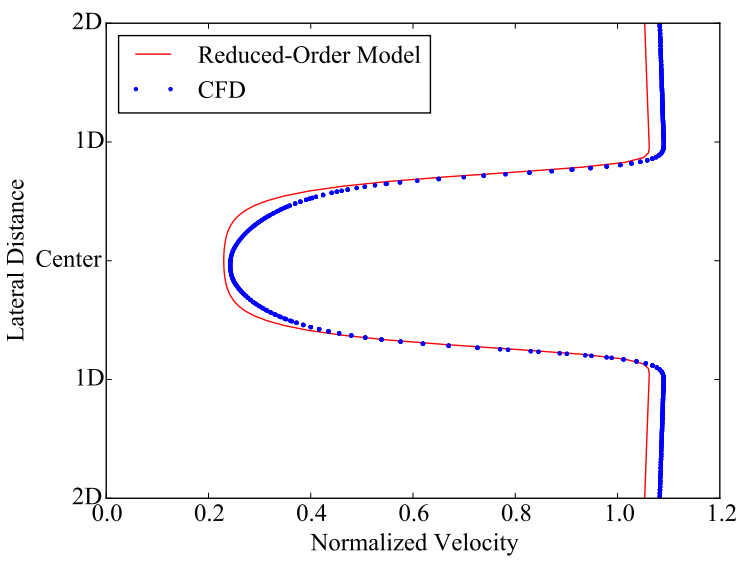

(d) $x / D=8.0 ; 5.29 \%$

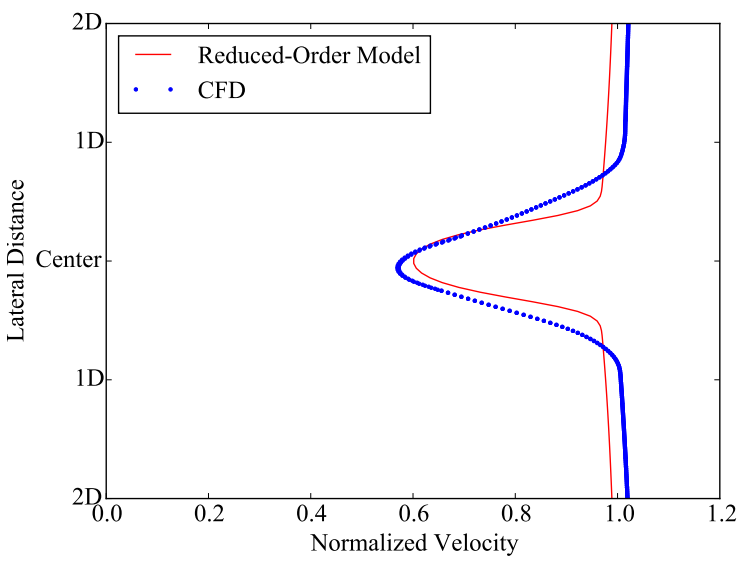

(f) $x / D=15.0 ; 5.62 \%$

Figure 8. The velocity profiles of the CFD data and the reduced-order model split up by the normalized downstream distance. Percent error of the maximum velocity deficit between the CFD and reduced-order model is also indicated. 
The reduced-order model was also compared to the experimental PIV study conducted by Tescione et al. ${ }^{20}$ The velocity profiles of the PIV, CFD, and reduced-order models were overlaid and can be seen in Fig. 9. The reduced-order wake model was created based only on the CFD data using the specifications of Kjellin et al., ${ }^{27}$ which demonstrates the wide applicability of the wake model. Looking at the velocity deficits, the reduced-order model predicted the maximum velocity deficit with a percent error of $19.6 \%$ at $x / D=2.0$ varying slightly from the CFD, as described above, which had $23.0 \%$ at this same location. The reduced-order model also matched the shape of the CFD data rather than the significant asymmetry of the PIV data.
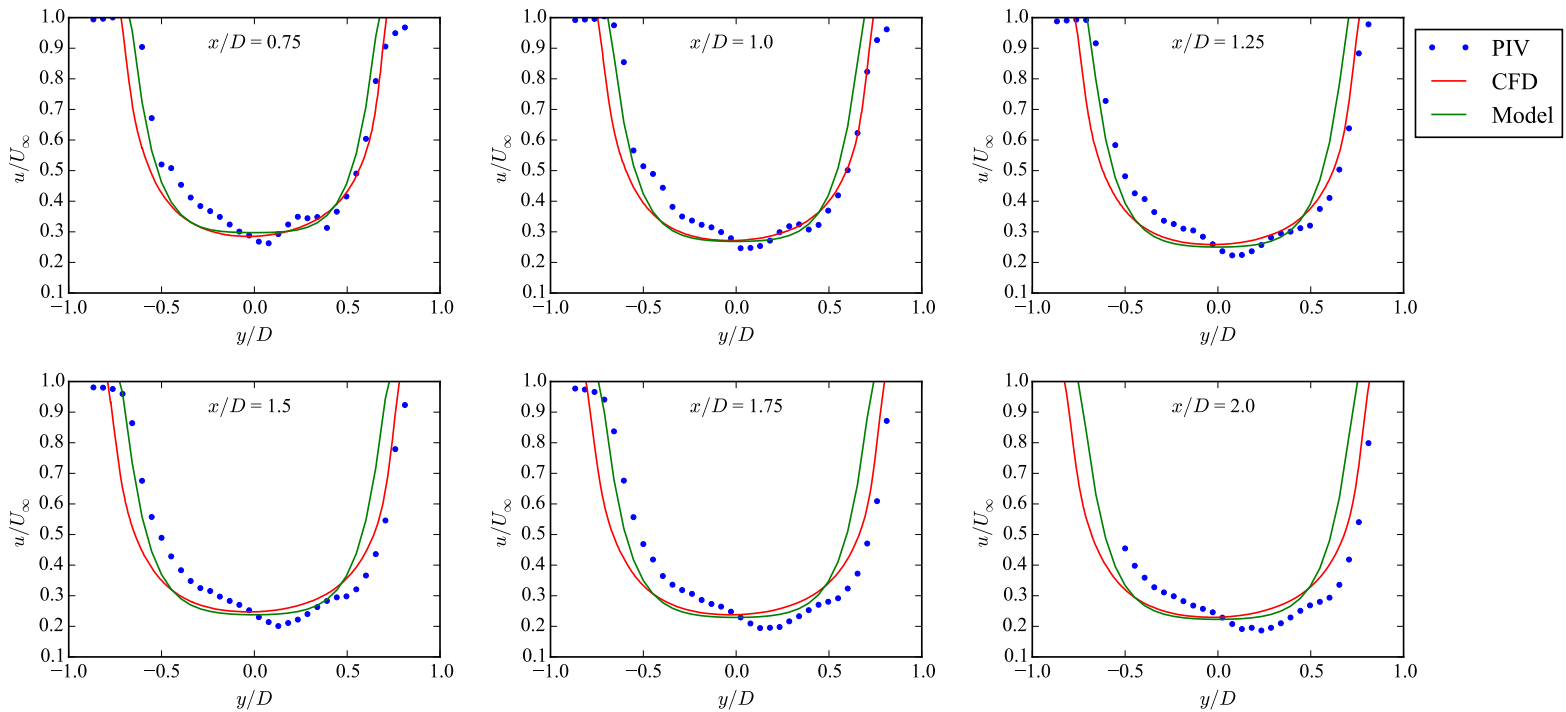

Figure 9. The wake velocity comparison seen in Fig. 2 with our reduced-order model based on the CFD model added.

For further validation, we also compared our reduced-order model's results to a wind tunnel study conducted by Battisti et al. ${ }^{28}$ In this study, a three-bladed VAWT with a 1.03 meter diameter was placed in a wind tunnel with a wind speed of about $16 \mathrm{~m} / \mathrm{s}$ resulting in a Reynolds number of about 1,000,000. The wind tunnel had a removable test room in which the VAWT could be placed and tests were run in both a closed configuration (the test room enclosed over the VAWT) and an open configuration (the test room removed and the VAWT open to the outside air). As VAWTs are generally operated in the open, we decided that the open configuration would be the best data to compare to our wake model. The wake velocity was recorded 1.5 diameters downstream of the turbine.

Extrapolation of our reduced-order model was needed as this test was run at a TSR of 1.6, outside of the range of CFD models tested. However, as seen in Fig. 10, our wake model matched well with the experimental results with a percent error of $11.2 \%$ of the experimental data. Extrapolation proved to produce reasonable results in this case, but we plan to continue expanding our CFD wake data to include TSRs lower than 2.5 to match this type of experimentation better. From these two validations, we can see that our reduced-order wake model gives reasonable velocity predictions that match well with experimental results. 


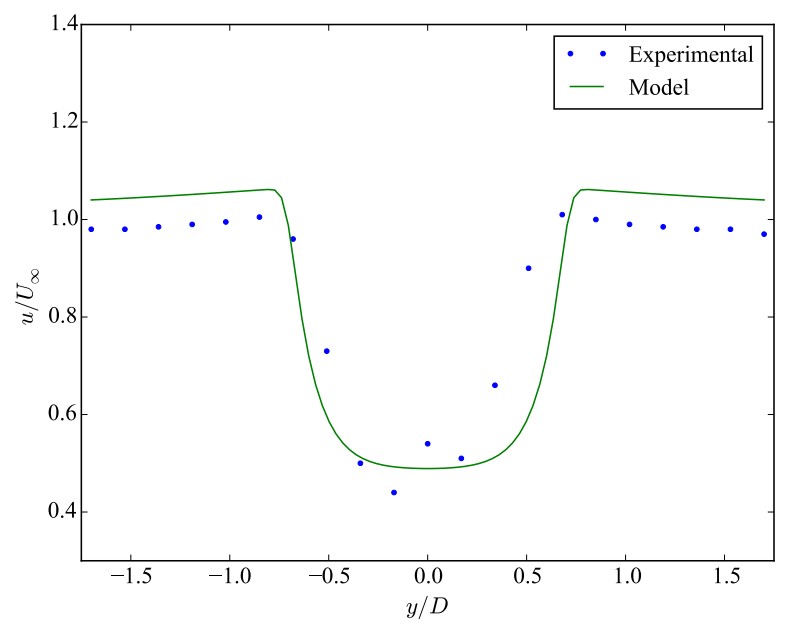

Figure 10. The wake velocity comparison between the wind tunnel study conducted by Battisti et al. ${ }^{28}$ and our reduced-order model. The velocity $(u)$ is normalized by the free stream wind speed $\left(U_{\infty}\right)$ and the lateral $(y)$ position is normalized by the turbine diameter $(D)$. This velocity profile was taken at 1.5 diameters downstream.

\section{Conclusion}

Using CFD data at different TSRs and solidities, we were able to compile large amounts of VAWT wake data which we parameterized with an EMG distribution and used to create a reduced-order model. The velocity profiles that the model predicted showed good agreement with the validated CFD data as well as PIV experimental data from Tescione et al. ${ }^{20}$ and wind tunnel experimental data from Battisti et al. ${ }^{28}$ These validation studies show, from an initial standpoint, that our reduced-order model provides accurate results much more quickly than experimental or CFD analysis for Reynolds numbers between about 180,000 and $6,000,000$. It also has the potential of producing effective results in large-scale wind turbine analysis. For about a 5-6\% accuracy loss compared to the CFD data, our reduced-order model produced velocity deficit results at any location in milliseconds for what would take a CFD simulation about a day to compute.

However, the model's ability to account for multiple wake interactions still needs to be developed. When multiple wakes interact, the velocity distribution behind the turbines is affected by the interference of wind momentum changes. As wind farms generally have many turbines, this wake interaction must be considered in order to ensure that velocity deficits in the wake regions are modeled accurately.

We plan to add this ability to our reduced-order model by looking at how other researchers have modeled wind turbine wake interaction. One study conducted by Gebraad et al. ${ }^{14}$ studied the wake interaction of HAWTs and used the sum of the squares of wake velocity deficits to compute an effective wake velocity deficit. We have also considered using an actuator cylinder method to calculate the velocity deficits at discrete points around the turbine based on the interacting wakes affecting the turbine, as demonstrated by Ning. ${ }^{29}$ Ultimately, we will produce a reduced-order wake model that will be used in wind farm layout optimization of VAWTs.

Originally we decided to use the vorticity as a means of calculating the wake velocity deficit due to the concentrated streams of vorticity that decayed downstream. We realized, however, that the vorticity data needed to be captured by means of trend fit analysis to produce accurate results. Because of this realization, we are looking into the possibility of capturing the velocity data directly with a form of distribution fitting. By doing this, the method of calculating the wake velocity deficit will be simpler and more intuitive instead of using complex calculations of the vorticity data. It will also allow us to model the slight asymmetry found in the CFD data easier which could be important in rotation direction analysis. We plan to study this method in future iterations of the parametric wake model. The VAWT CAD models used in the CFD simulations, the CFD wake data and the EMG parameters at each TSR and solidity, and a working parameterized wake model can be accessed at: http://flow.byu.edu/publications/ 


\section{References}

${ }^{1}$ Bazilevs, Y., Korobenko, A., Deng, X., Yan, J., Kinzel, M., and Dabiri, J. O., "Fluid-Structure Interaction Modeling of Vertical-Axis Wind Turbines," Journal of Applied Mechanics, Vol. 81, August 2014.

${ }^{2}$ Fichaux, N., Wilkes, J., Hulle, F. V., and Cronin, A., "Oceans of Opportunity: Harnessing Europe's largest domestic energy resource," Tech. Rep. 41018647, European Wind Energy Association, September 2009.

${ }^{3}$ Maples, B., Saur, G., Hand, M., van de Pietermen, R., and Obdam, T., "Installation, Operation, and Maintenance Strategies to Reduce the Cost of Offshore Wind Energy," Tech. Rep. NREL/TP-5000-57403, National Renewable Energy Laboratory, July 2013.

${ }^{4}$ Dabiri, J. O., Greer, J. R., Koseff, J. R., Moin, P., and Peng, J., "A New Approach To Wind Energy: Opportunities and Challenges," AIP Conference Proceedings, 2015.

${ }^{5}$ Sandia National Laboratories, "Offshore use of vertical-axis wind turbines gets closer look," July 2012.

${ }^{6}$ Sutherland, H. J., Berg, D. E., and Ashwill, T. D., "A Retrospective of VAWT Technology," Tech. rep., Sandia National Laboratories, January 2012.

${ }^{7}$ Vermeer, L., Sørensen, J., and Crespo, A., "Wind turbine wake aerodynamics," Progress in Aerospace Sciences, Vol. 39, 2003, pp. 467-510.

${ }^{8}$ Crespo, A., Hernández, J., and Frandsen, S., "Survey of Modelling Methods for Wind Turbine Wakes and Wind Farms," Wind Energy, Vol. 2, 1999, pp. 1-24.

${ }^{9}$ Sanderse, B., "Aerodynamics of wind turbine wakes," Tech. Rep. ECN-E09-016, Energy Centre of the Netherlands, 2009.

${ }^{10}$ Changshui, Z., Guangdong, H., and Jun, W., "A fast algorithm based on the submodular property for optimization of wind turbine positioning," Renewable Energy, Vol. 36, 2011, pp. 2951-2958.

${ }^{11}$ Kusiak, A. and Song, Z., "Design of wind farm layout for maximum wind energy capture," Renewable Energy, Vol. 35, No. 3, 2010, pp. 685-694.

${ }^{12}$ Eroğlu, Y. and Seçkiner, S. U., "Design of wind farm layout using ant colony algorithm," Renewable Energy, Vol. 44, 2014, pp. 53-62.

${ }^{13}$ Pérez, B., Mínguez, R., and Guanche, R., "Offshore Wind Farm Layout Optimization Using Mathematical Programming Techniques," Renewable Energy, Vol. 53, 2013, pp. 389-399.

${ }^{14}$ Gebraad, P. M. O., Teeuwisse, F. W., van Wingerden, J. W., Fleming, P. A., Ruben, S. D., Marden, J. R., and Pao, L. Y., "Wind plant power optimization through yaw control using a parametric model for wake effects-a CFD simulation study," Wind Energy, 2014.

${ }^{15}$ Gebraad, P. M., Thomas, J. J., Ning, A., Fleming, P. A., and Dykes, K., "Maximization of the annual energy production of wind power plants by optimization of layout and yaw-based wake control," (in review).

${ }^{16}$ Fleming, P., Ning, A., Gebraad, P., and Dykes, K., "Wind Plant System Engineering through Optimization of Layout and Yaw Control," Wind Energy, March 2015.

${ }^{17}$ Sheldahl, R. E. and Klimas, P. C., "Aerodynamic Characteristics of Seven Symmetrical Airfoil Sections Through 180Degree Angle of Attack for Use in Aerodynamic Analysis of Vertical Axis Wind Turbines," Tech. rep., Sandia National Laboratories, Advanced Energy Projects Division 4715, Sandia National Laboratories, Albuquerque, NM 87185, March 1981.

${ }^{18}$ Sheldahl, R. E., "Comparison of Field and Wind Tunnel Darrieus Wind Turbine Data," Tech. rep., Sandia National Laboratories, January 1981.

${ }^{19}$ Worstell, M. H., "Aerodynamic Performance of the 17 Meter Diameter Darrieus Wind Turbine," Tech. rep., Sandia National Laboratories, September 1978.

${ }^{20}$ Tescione, G., Ragni, D., He, C., Ferreira, C. S., and van Bussel, G., "Near wake flow analysis of a vertical axis wind turbine by stereoscopic particle image velocimetry," Renewable Energy, Vol. 70, 2014, pp. 47-61.

${ }^{21}$ Ferreira, C. J. S., The Near Wake of the VAWT: $2 D$ and $3 D$ Views of the VAWT Aerodynamics, Ph.D. thesis, Delft University of Technology, 2009.

${ }^{22}$ Ferreira, C. S., Madsen, H. A., Barone, M., Roscher, B., Deglaire, P., and Arduin, I., "Comparison of aerodynamic model for Vertical Axis Wind Turbines," Journal of Physics, Vol. 524, 2014.

${ }^{23} \mathrm{He}$, C., Wake Dynamics Study of an H-type Vertical Axis Wind Turbine, Master's thesis, Delft University of Technology, August 2013.

${ }^{24}$ Dixon, K. R., The Near Wake Structure of a Vertical Axis Wind Turbine: Including the Development of a 3D Unsteady Free-Wake Panel Method for VAWTs, Master's thesis, Delft University of Technology, April 2008.

${ }^{25}$ Shamsoddin, S. and Porté-Agel, F., "Large Eddy Simulation of Vertical Axis Wind Turbine Wakes," Energies, Vol. 7, 2014, pp. 8990-8912.

${ }^{26}$ Whittlesey, R. W., Liska, S., and Dabiri, J. O., "Fish schooling as a basis for vertical axis wind turbine farm design," Tech. rep., California Institute of Technology, February 2010.

${ }^{27}$ Kjellin, J., Bülow, F., Eriksson, S., Deglaire, P., Leijon, M., and Bernhoff, H., "Power coefficient measurement on a 12 kW straight bladed vertical axis wind turbine," Renewable Energy, Vol. 36, 2011, pp. 3050-3053.

${ }^{28}$ Battisti, L., Zanne, L., Dell'Anna, S., Dossena, V., Persico, G., and Paradiso, B., "Aerodynamic Measurements on a Vertical Axis Wind Turbine in a Large Scale Wind Tunnel," Journal of Energy Resources Technology, Vol. 133, September 2011.

${ }^{29}$ Ning, A., "Actuator Cylinder Theory for Multiple Vertical Axis Wind Turbines," (in review), 2015. 